Biochimica et Biophysica Acta, 406 (1975) 97-107

(C) Elsevier Scientific Publishing Company, Amsterdam - Printed in the Netherlands

BBA 77063

\title{
RELATION BETWEEN VARIOUS PHOSPHOLIPASE ACTIONS ON HUMAN RED CELL MEMBRANES AND THE INTERFACIAL PHOSPHOLIPID PRES- SURE IN MONOLAYERS
}

R. A. DEMEL, W. S. M. GEURTS VAN KESSEL, R. F. A. ZWAAL, B. ROELOFSEN and L. L. M. VAN DEENEN

Laboratory of Biochemistry, State University of Utrecht, Transitorium 3, University Centre "De Uithof" Padualaan 8, Utrecht (The Netherlands)

(Received April 18th, 1975)

\section{SUMMARY}

The action of purified phospholipases on monomolecular films of various interfacial pressures is compared with the action on erythrocyte membranes. The phospholipases which cannot hydrolyse phospholipids of the intact erythrocyte membrane, phospholipase $\mathrm{C}$ from Bacillus cereus, phospholipase $\mathrm{A}_{2}$ from pig pancreas and Crotalus adamanteus and phospholipase D from cabbage, can hydrolyse phospholipid monolayers at pressure below 31 dynes/cm only.

The phospholipases which can hydrolyse phospholipids of the intact erythrocyte membrane, phospholipase $\mathrm{C}$ from Clostridium welchii phospholipase $\mathrm{A}_{2}$ from Naja naja and bee venom and sphingomyelinase from Staphylococcus aureus, can hydrolyse phospholipid monolayers at pressure above $31 \mathrm{dynes} / \mathrm{cm}$. It is concluded that the lipid packing in the outer monolayer of the erythrocyte membrane is comparable with a lateral surface pressure between 31 and 34.8 dynes $/ \mathrm{cm}$.

\section{INTRODUCTION}

Phospholipases from different sources have been used to reveal information regarding the localization of the lipids within the erythrocyte membrane [1-4]. These phospholipases show a great variety of behaviour against biological membranes and model systems. Some phospholipases can hydrolyse the phospholipids of the intact erythrocyte membrane which can lead to hemolysis of the cell. Other phospholipases cannot hydrolyse the phospholipids of the intact cell but can hydrolyse the phospholipids of ghost membranes [4]. Questions as why only some phospholipases can degrade the phospholipids of intact cells are difficult to answer from studies on biological membranes. It would be of interest to know if the phospholipases have different abilities to penetrate the membrane or if the lipid composition could play a role. Monolayers have shown to be a useful tool in the study of interfacial properties of phospholipids and to study enzymatic hydrolysis [5-8]. The 
molecular packing of lipids can be changed by compressing the monolayer to a different extent. The monolayer composition can be varied to a large extent by the use of synthetic and isolated lipids. The enzymatic degradation by phospholipase $C$ can be easily followed by detecting the surface radioactivity of a methyl-labelled phospholipid. In this paper the effects of surface pressure and lipid composition are examined for different phospholipases. The results on monomolecular films are compared with those on intact erythrocyte membranes [4].

\section{MATERIALS AND METHODS}

The following phospholipases were purified as described in the preceding paper [4]. Phospholipase $\mathrm{A}_{2}$ from porcine pancreas, phospholipase $\mathrm{A}_{2}$ from bee venom (Apis mellifica), phospholipase $\mathrm{A}_{2}$ from $N$ aja naja, phospholipase $\mathrm{A}_{2}$ from Crotalus admanteus, phospholipase $\mathrm{C}$ from Bacillus cereus, phospholipase $\mathrm{C}$ from Clostridium welchii, sphingomyelinase C from Staphylococcus aureus and phospholipase D from savoy cabbage. In 1-palmitoyl-2-oleoyl-sn-glycero-3-phosphatidylcholine a ${ }^{14} \mathrm{C}$-labelled methyl group was introduced into the choline moiety according to the method of Stoffel et al. [9]. Sphingomyelin from pig brain was labelled by the same procedure.

Phosphatidylserine was isolated from pig brain. All phospholipids used were chromatographically pure. The phospholipase $\mathrm{C}$ from $B$. cereus and $C$. welchii, sphingomyelinase $\mathrm{C}$ and phospholipase $\mathrm{D}$ action on phospholipid monolayers was followed by a simultaneous recording of the surface pressure and surface radioactivity during the experiment. Measurements were performed at $37^{\circ} \mathrm{C}$ in a thermostated box. Monolayers were spread in a circular trough $8 \mathrm{~cm}$ in diameter and $3 \mathrm{~cm}$ deep. The trough was filled with $10^{-2} \mathrm{M}$ Tris-acetate buffer $\mathrm{pH} 7.4$. In the case of phospholipase $\mathrm{A}_{2}$ from $N$. naja and phospholipase $\mathrm{D}$ the buffer contained $10 \mathrm{mM}$ $\mathrm{CaCl}_{2}$. The $\mathrm{Ca}^{2+}$ requirement of the other phospholipases $\mathrm{A}_{2}$ was met by the addition of $1 \mathrm{mM} \mathrm{CaCl}$. In the case of phospholipase $\mathrm{C}$ from $C$. welchii the buffer contained $1 \mathrm{mM} \mathrm{CaCl}_{2}$ and $0.1 \mathrm{mM} \mathrm{ZnCl}_{2}$. In the case of sphingomyelinase from $S$. aureus $0.25 \mathrm{mM} \mathrm{MgCl}$ was added. For phospholipase $\mathrm{C}$ from $B$. cereus the $\mathrm{Ca}^{2+}$, bound to the enzyme, was sufficient for the enzymatic hydrolyses. The phospholipase $\mathrm{A}_{2}$ action on long chain phospholipids was also measured in the way described above. The action of phospholipases on a short chain phosphatidylcholine, dinonanoyl phosphatidylcholine, was measured with the zero order trough as described by Verger and de Haas [8].

\section{RESULTS}

At the concentration used, the pure phospholipases showed no surface activity when injected in the subphase. The effect of the molecular packing of ${ }^{14} \mathrm{C}$ labelled palmitoyl-oleoyl phosphatidylcholine on the phospholipase $\mathrm{C}$ degradation is shown in Fig. 1. Fig. $1 \mathrm{~A}$ and $\mathrm{B}$ show the actual recordings of the degradation. At a pressure of 29.4 dynes/cm a rapid release of the phosphate-choline moiety is measured by the decrease in radioactivity, after a lack period of $4 \mathrm{~min}$. The degradation is completed in about 12 min (Fig. 1A). The simultaneous measurement of the surface pressure shows a decrease due to the transformation of phosphatidylcholine 

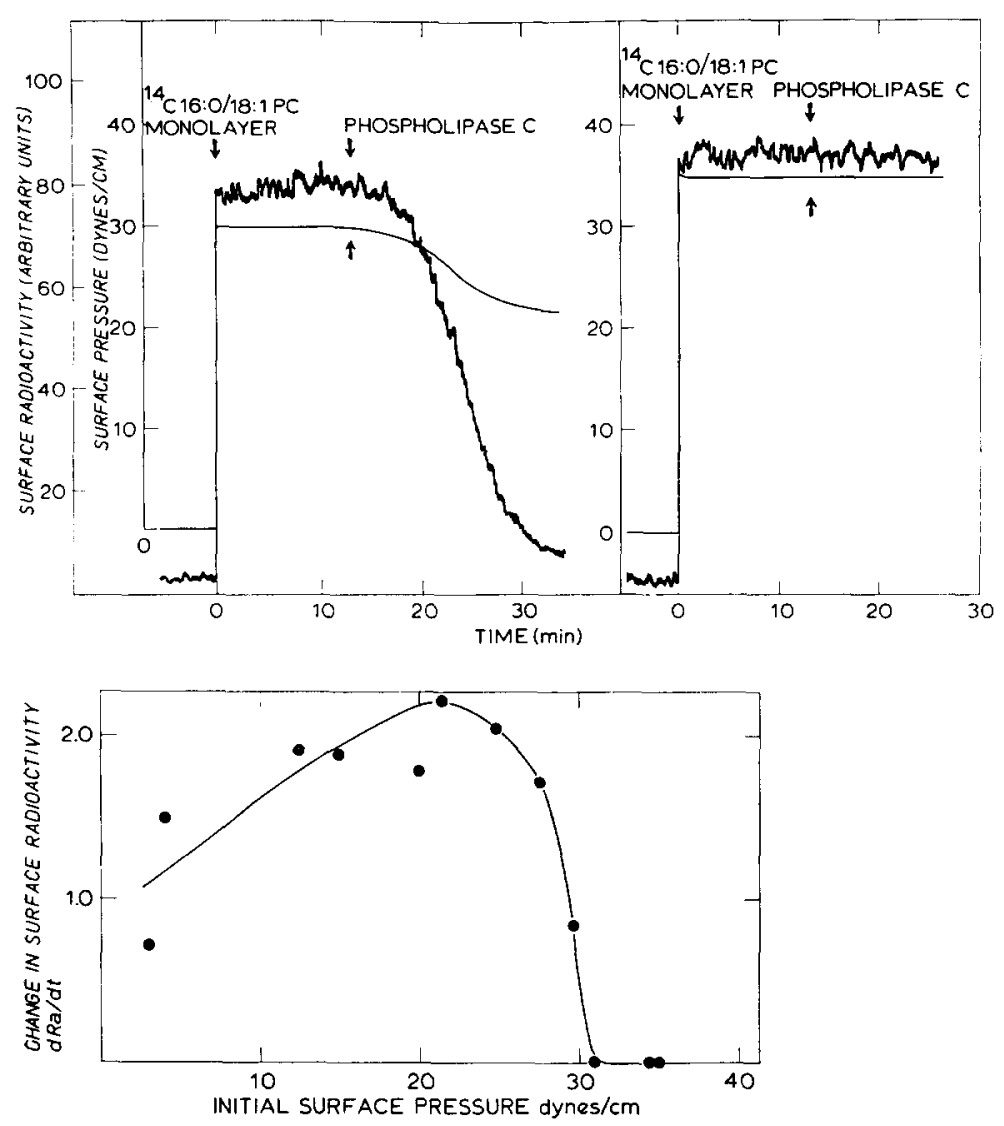

Fig. 1. A (left) and B (right). Change in surface radioactivity (Ra) and surface pressure of ${ }^{14} \mathrm{C}$. labelled palmitoyl-oleoyl phosphatidylcholine monolayers upon hydrolysis by phospholipase $\mathrm{C}$ from B. cereus (0.16 I.U.). The subsolution consists of $10^{-2} \mathrm{M}$ Tris pH 7.4 at $37^{\circ} \mathrm{C}$. C. Relation between the change in surface radioactivity and the initial surface pressure of ${ }^{14} \mathrm{C}$-labelled palmitoyl-oleoyl phosphatidylcholine monolayers upon hydrolyses by phospholipase C from $B$. cereus (0.16 I.U.).

to diacylglycerol. At an initial surface pressure of 34.8 dynes $/ \mathrm{cm}$ no phosphatidylcholine degradation can be measured even when additional amounts of phospholipase $\mathrm{C}$ are injected (Fig. 1B). A detailed surface pressure dependance (Fig. 1C) shows a rapid hydrolysis of palmitoyl-oleoyl phosphatidylcholine at pressures below 30 dynes $/ \mathrm{cm}$. Above 31 dynes $/ \mathrm{cm}$ hydrolysis stops completely. Sphingomyelin cannot be degraded by phospholipase $\mathrm{C}$ from $\boldsymbol{B}$. cereus. For the human erythrocyte it was demonstrated that the outer layer consists mainly of phosphatidylcholine and sphingomyelin [1]. The phospholipase $C$ from $B$. cereus does not produce phospholipid degradation in these intact cells. It could be questioned if sphingomyelin would affect phosphatidylcholine degradation by phospholipase $C$ from $B$. cereus. Therefore, the phospholipase $C(B$. cereus) degradation of mixed monolayers of phosphatidylcholine and sphingomyelin (molar ratio 65:35) is studied (Fig. 2). The molar ratio used is close to the ratio found in the mammalian erythrocyte membrane. 


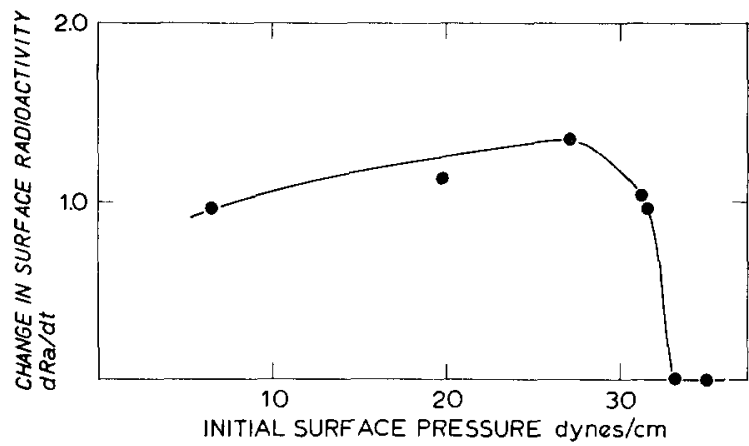

Fig. 2. Relation between the change in surface radioactivity and the initial surface pressure of mixed monolayers of ${ }^{14} \mathrm{C}$-labelled palmitoyl-oleoyl phosphatidylcholine and sphingomyelin, molar ratio $65: 35$, upon hydrolyses by phospholipase $\mathrm{C}$ from B. cereus. (0.16 I.U.). The subsolution consists of $10^{-2} \mathrm{M}$ Tris $\mathrm{pH} 7.4$ at $37^{\circ} \mathrm{C}$.

The rate of radioactivity change is somewhat reduced compared to the pure phosphatidylcholine film (Fig. 1) due to substrate dilution. The maximum surface pressure at which degradation is still possible is only slightly affected. Above pressures of 33 dynes/cm no enzyme activity is found. As described in the preceding paper [4] phospholipase $\mathrm{C}$ was found to hydrolyse phosphatidylcholine in erythrocyte membranes when the cell was pretreated with sphingomyelinase from $S$. aureus. Fig. 3 shows that when the sphingomyelinase activity ( $S$. aureus) is tested upon ${ }^{14} \mathrm{C}$ methyl-labelled sphingomyelin degradation is possible at all surface pressures obtainable. Also in mixed monolayers formed of phosphatidylcholine and sphingomyelin (molar ratio 65:35) sphingomyelin is converted to ceramide by sphingomyelinase ( $S$. aureus) at all monolayer pressures even at pressures reaching the collapse pressure of the monolayer. Sphingomyelinase from $S$. aureus cannot degrade phosphatidylcholines. When this sphingomyelinase is injected underneath a mixed monolayer of phosphatidylcholine and sphingomyelin of $42 \mathrm{dynes} / \mathrm{cm}$ the pressure decreases to 37.5 dynes/cm due to the formation of ceramide (Fig. 4). At this pressure phospholipase $C$ (B. cereus) is not able to degrade a pure phosphatidylcholine film (Fig. 1) or a mixed monolayer of phosphatidylcholine and sphingomyelin (Fig. 2). However,

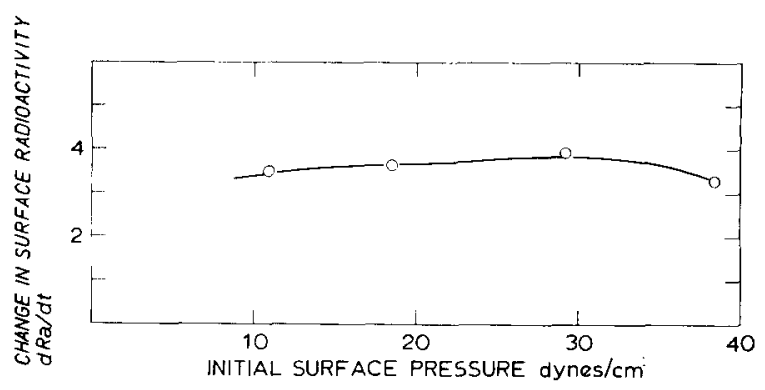

Fig. 3. Relation between the change in surface radioactivity and the initial surface pressure of ${ }^{14} \mathrm{C}$ labelled sphingomyelin monolayers upon hydrolysis by sphingomyelinase $\mathrm{C}$ from $S$. aureus $(0.16$ J.U.). The subsolution consists of $10^{-2} \mathrm{M}$ Tris and $0.25 \mathrm{mM} \mathrm{MgCl}_{2}, \mathrm{pH} 7.4$ at $37{ }^{\circ} \mathrm{C}$. 


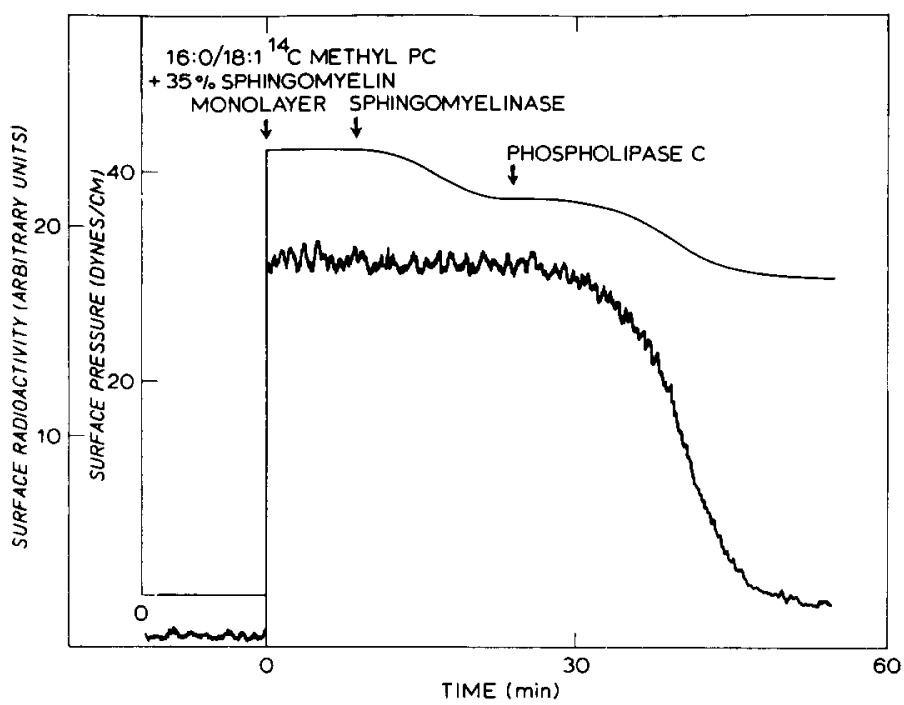

Fig. 4. Change in surface radioactivity and surface pressure of a mixed monolayer of ${ }^{14} \mathrm{C}$-labelled palmitoyl-oleoyl phosphatidylcholine (PC) and sphingomyelin, molar ratio $65: 35$, upon subsequent hydrolysis by sphingomyelinase $\mathrm{C}$ from $S$. aureus $(0.16 \mathrm{I}$.U.) and phospholipase $\mathrm{C}$ from $B$. cereus (0.16 I.U.). The subsolution consists of $10^{-2} \mathrm{M}$ Tris and $0.25 \mathrm{mM} \mathrm{MgCl}_{2}, \mathrm{pH} 7.4$ at $37^{\circ} \mathrm{C}$.

when phospholipase $\mathrm{C}$ (B. cereus) is injected underneath this film a rapid enzymatic degradation is observed by the change in surface radioactivity and surface pressure. The presence of ceramide formed by the sphingomyelinase treatment allows phospholipase $C$ (B. cereus) activity at pressures above 31 dynes $/ \mathrm{cm}$. The enzymatic degradation by phospholipase $C$ (B. cereus) at high surface pressures can also be achieved by other compounds such as diacylglycerol, negatively and positively charged compounds (Fig. 5). It can be seen that phosphatidylcholine monolayers

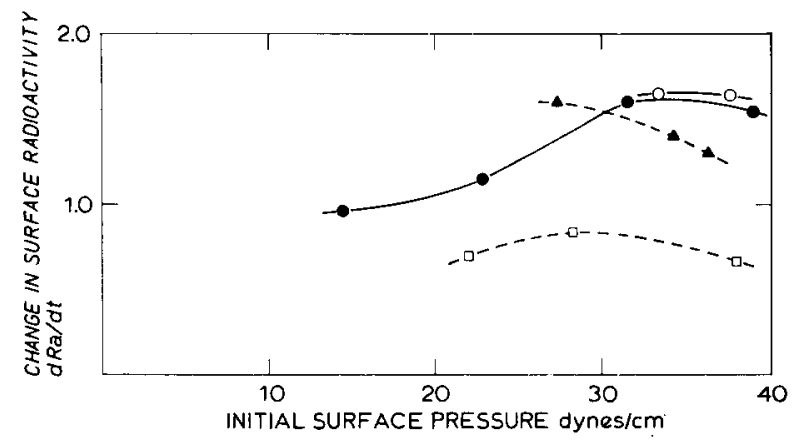

Fig. 5. Relation between the change in surface radioactivity and the initial surface pressure of mixed monolayers of respectively: ${ }^{14} \mathrm{C}$-labelled palmitoyl-oleoyl phosphatidylcholine and myristoyl-oleoyl diacylglycerol molar ratio $1: 1(0) ;{ }^{14} \mathrm{C}$-labelled palmitoyl-oleoyl phosphatidylcholine and ceramide, molar ratio $65: 35(\bigcirc) ;{ }^{14} \mathrm{C}$-labelled palmitoyl-oleoyl phosphatidylcholine and phosphatidic acid, molar ratio $80: 20(\Delta) ;{ }^{14} \mathrm{C}$-labelled palmitoyl-oleoyl phosphatidylcholine and stearylamine, molar ratio $80: 20(\square)$ upon hydrolysis by phospholipase $C$ from $B$. cereus $(0.16$ I.U.). The solution consists of $10^{-2} \mathrm{M}$ Tris, $\mathrm{pH} 7.4$ at $37^{\circ} \mathrm{C}$. 


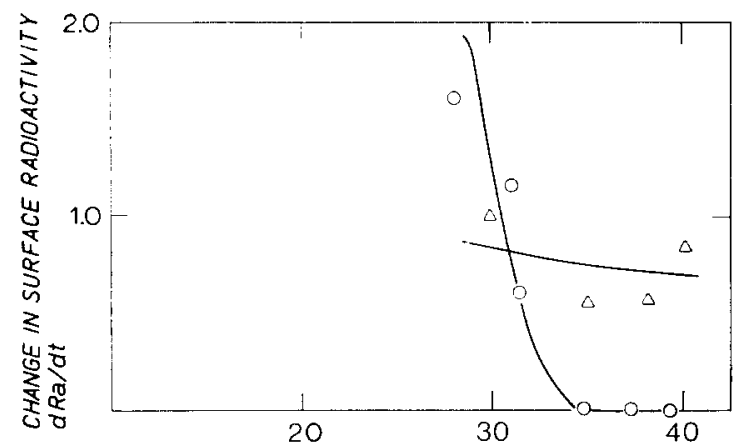

INITIAL SURFACE PRESSURE dynes/cm

Fig. 6. Relation between the change in surface radioactivity and the initial surface pressure of mixed monolayers of respectively: ${ }^{14} \mathrm{C}$-labelled palmitoyl-oleoyl phosphatidylcholine, sphingomyelin and cholesterol, molar ratio $15: 12: 9(\bigcirc) ;{ }^{14} \mathrm{C}$-labelled palmitoyl-oleoyl phosphatidylcholine, sphingomyelin and cholesterol $15: 12: 25(\triangle)$ upon hydrolysis by phospholipase $C$ from $B$. cereus $(0.16$ I.U.). The subsolution consists of $10^{-2} \mathrm{M}$ Tris, pH 7.4 at $37^{\circ} \mathrm{C}$.

containing ceramide (prepared from sheep erythrocyte sphingomyelin) and myristoyloleoyl diacylglycerol both enhance phosphatidylcholine degradation at pressures above 31 dynes $/ \mathrm{cm}$. The presence of $20 \mathrm{~mol} \%$ phosphatidic acid shows a marked enhancement of enzymatic activity at higher pressures. The positively charged stearylamine also shows stimulation, but to a lesser extent. Low concentrations of cholesterol (molar ratio phosphatidylcholine/sphingomyelin/cholesterol 15:12:9) do not show much enhancement of the phospholipase C (B. cereus) activity (Fig. 6). At molar ratios of phosphatidylcholine/sphingomyelin/cholesterol $(15: 12: 25)$, phosphatidylcholine breakdown can be detected at high pressures. Phospholipase $\mathrm{C}$ from C. welchii can hydrolyse the phospholipids and lyse the erythrocyte cell membrane [4]. The phospholipase C from $C$. welchii can degrade phosphatidylcholine as well as sphingomyelin. The degradation of ${ }^{14} \mathrm{C}$-labelled palmitoyl-oleoyl phosphatidylcholine, by phospholipase $\mathrm{C}$ from $C$. welchii proceeds at a lower pressure (28 dynes/ $\mathrm{cm}$ ) (Fig. 7) than by phospholipase $\mathrm{C}$ from B. cereus (Fig. 1C). However, the degradation of ${ }^{14} \mathrm{C}$-labelled sphingomyelin is possible at all surface pressures, although

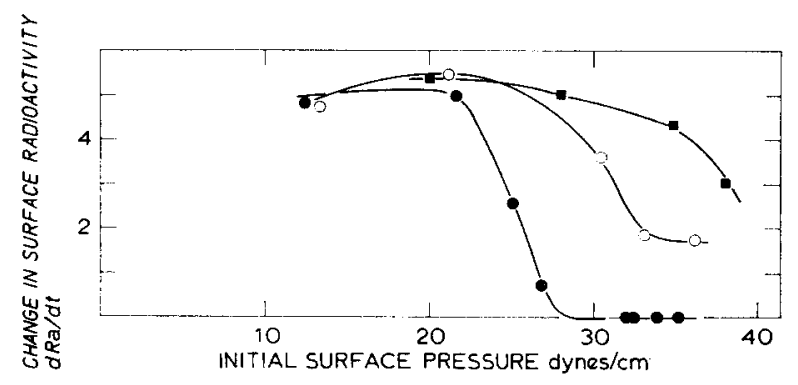

Fig. 7. Relation between the change in surface radioactivity and initial surface pressure of the following monolayers: ${ }^{14} \mathrm{C}$-labelled palmitoyl-oleoyl phosphatidylcholine $(O) ;{ }^{14} \mathrm{C}$-labelled sphingomyelin $(\bigcirc) ;{ }^{14} \mathrm{C}$-labelled palmitoyl-oleoyl phosphatidylcholine sphingomyelin/cholesterol molar ratio $15: 12: 9$ (ם) upon hydrolysis by phospholipase $\mathrm{C}$ from $C$. welchii $(0.16 \mathrm{I}$.U.). The subsolution consists of $10^{-2} \mathrm{M}$ Tris, $1 \mathrm{~m} \mathrm{M} \mathrm{CaCl}_{2}, 0.1 \mathrm{mM} \mathrm{ZnCl}_{2}, \mathrm{pH} 7.4$ at $37^{\circ} \mathrm{C}$. 
the rate is reduced at high surface pressures. In contrast to phospholipase $\mathrm{C}$ from $B$. cereus the phospholipase $\mathrm{C}$ from $C$. welchii degrades mixtures of phosphatidylcholine/sphingomyelin/cholesterol $(15: 12: 9)$ up to the highest pressure attainable (Fig. 7). This is also the case for mixtures of phosphatidylcholine/sphingomyelin/ cholesterol (15:12:25).

Some of the phospholipases $\mathrm{A}_{2}$ have been shown to hydrolyse the phospholipids of the erythrocyte membrane whereas phospholipases $A_{2}$ from other sources do not. The enzymatic activity of phospholipases $\mathrm{A}_{2}$ cannot be followed accurately by surface radioactivity as is the case with phospholipase $C$. The degradation products of phospholipase $\mathrm{A}_{2}$ activity on physiological phosphatidylcholines, fatty acids and lysophosphatidylcholines are not readily water soluble. The departure from the interface is dependent on the surface pressure and on the collapse pressure of the products. The rate of enzymatic degradation can be accurately measured with the zero order trough as described by Verger and de Haas [8], with dinonanoyl phosphatidylcholine as substrate. The reaction products of this short chain phosphatidylcholine are water soluble.

Phospholipases $\mathrm{A}_{2}$ from pig pancreas and from $\mathrm{Cr}$. adamanteus are not able to attack intact human erythrocytes. Fig. $8 \mathrm{~A}$ and $\mathrm{B}$ shows that phospholipase $\mathrm{A}_{2}$ enzymatic activity from pig pancreas and from $\mathrm{Cr}$. adamanteus stops at pressures of 16.2 and 23.3 dynes $/ \mathrm{cm}$ respectively when dinonanoyl phosphatidylcholine is used as a substrate. Injection of higher amounts of these phospholipases does not result in an enzymatic activity at higher pressures. To ensure that the maximum pressure

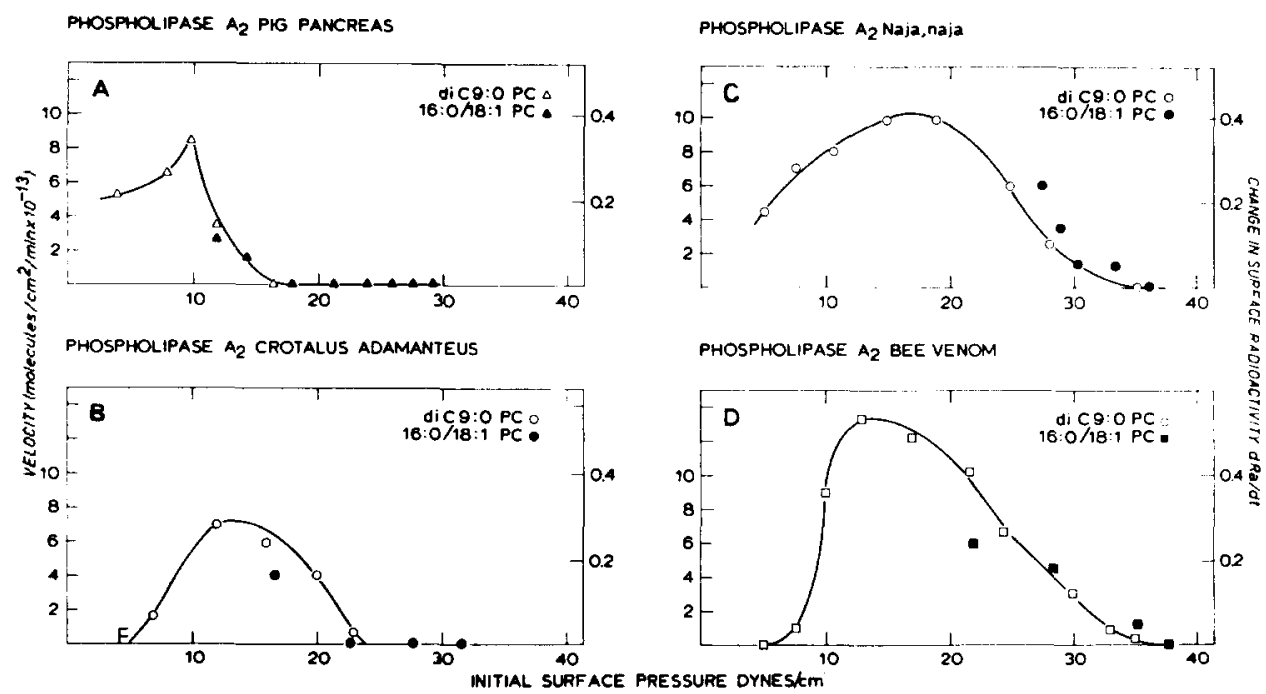

Fig. 8. Relation between the degradation velocity and initial surface pressure of dinonanoyl phos. phatidylcholine (measured by the change in surface area [8]) and ${ }^{14} \mathrm{C}$-labelled palmitoyl-oleoyl phosphatidylcholine (measured by the change in surface radioactivity), upon hydrolysis by respectively: phospholipase $A_{2}$ from pig pancreas (A); phospholipase $A_{2}$ from $C_{r}$. adamanteus (B); phospholipase $\mathrm{A}_{2}$ from $N$. naja (C); phospholipase $\mathrm{A}_{2}$ from bee venom (D) $(0.20$ I.U.). The subsolution consists of $10^{-2} \mathrm{M}$ Tris, $1 \mathrm{mM} \mathrm{CaCl} 2, \mathrm{pH} 7.4$ at $37^{\circ} \mathrm{C}$. In the case of phospholipase $\mathrm{A}_{2}$ from $\mathrm{N}$. naja, the buffer contained also $10 \mathrm{mM} \mathrm{CaCl}{ }_{2}$. PC. phosphatidylcholine. 
measured for dinonanoyl phosphatidylcholine is also valid for a physiological phosphatidylcholine, some qualitative measurements were done with palmitoyl-oleoyl phosphatidylcholine as substrate. Measurements of the change of the initial surface radioactivity show indeed a practically identical pressure dependance for these two phosphatidylcholines. Phospholipases $\mathrm{A}_{2}$ from $N$. naja and bee venom can attack the phospholipids of intact human erythrocytes. When the enzymatic activity of phospholipase $\mathrm{A}_{2}$ from $N$. naja and bee venom is tested towards dinonanoyl phosphatidylcholine monolayers it can be demonstrated that the enzymatic activity is retained up to surface pressures of 34.8 and 35.3 dynes $/ \mathrm{cm}$, respectively (Fig. 8C and D). Corresponding behaviour is found when palmitoyl-oleoyl phosphatidylcholine is used as a substrate measuring the change in initial surface radioactivity. In the above experiments the enzymatic activity of phospholipases on monolayers was compared with the activity towards intact erythrocytes. Phospholipase $\mathrm{C}$ from $B$. cereus and phospholipase $\mathrm{A}_{2}$ from pig pancreas are not able to attack the intact erythrocyte membrane. However, when trapped in resealed erythrocyte ghosts, these two phospholipases are also able to hydrolyse the phospholipids of the inner membrane $[4,10]$. It has been shown that the outer membrane surface of the erythrocyte consists mainly of phosphatidylcholine and sphingomyelin whereas the inner membrane surface consists of phosphatidylethanolamine and phosphatidylserine as the most prominent phospholipids. The difference in hydrolysis of phospholipids as present in the outer and inner surface are tested in monomolecular films. Monomolecular films of dioleoyl phosphatidylethanolamine/phosphatidylserine, molecular ratio $2: 1$, are hydrolysed by pig pancreas phospholipase $A_{2}$ at pressure of even 39 dynes $/ \mathrm{cm}$. Also, a similar mixture of dioleoyl phosphatidylcholine/phosphatidylserine showed to be readily converted at pressures of 36 dynes $/ \mathrm{cm}$. Pure films of dimyristoyl phosphatidylglycerol/phosphatidylserine are also hydrolysed at high pressures. Hydrolysis by pig pancreas phospholipase $\mathrm{A}_{2}$ of dioleoyl phosphatidylcholine proceeds at pressures below 20 dynes $/ \mathrm{cm}$.

Phospholipase $C$ from $B$. cereus, only able to attack the inner erythrocyte membrane surface, hydrolyses mixtures of dioleoyl phosphatidylethanolamine/ phosphatidylserine and dioleoyl phosphatidylcholine/phosphatidylserine (molar ratio $2: 1$ ) at pressures of 39.4 and 37.0 dynes $/ \mathrm{cm}$, respectively. A pure film of phosphatidylserine is hydrolysed at 39.6 dynes $/ \mathrm{cm}$. The degradation of dioleoyl phosphatidylethanolamine by phospholipase C from $B$. cereus is practically the same as the respective phosphatidylcholine. Phospholipase D from cabbage, which does not produce hydrolysis of phospholipids in the intact cell, shows in monomolecular films of palmitoyl-oleoyl phosphatidylcholine an activity only at pressures below 20.5 dynes $/ \mathrm{cm}$.

\section{DISCUSSION}

Many investigators have already shown the use of monolayers in the study of phospholipase action. In most cases the enzymes were not of a defined purity to correlate the monolayer action and the degradation of phospholipids in a biological membrane.

As mentioned already, phospholipase $\mathrm{C}$ from $B$. cereus cannot attack the phospholipids of the intact cell. The monolayer studies show that palmitoyl-oleoyl 
phosphatidylcholine with interfacial pressures of more than 31 dynes $/ \mathrm{cm}$ is not broken down (Fig. 1C). The maximum enzymatic activity is between pressures of 27 and 12 dynes $/ \mathrm{cm}$. The reduced activities at pressures below 10 dynes $/ \mathrm{cm}$ can be attributed to surface denaturation as was found for phospholipase from C. welchii [7]. For palmitoyl-oleoyl phosphatidylcholine, the critical interfacial pressure of 31 dynes/ $\mathrm{cm}$ corresponds with a molecular area of $60 \AA^{2} / \mathrm{mol}$. The same molecular area was found to be critical for dimyristoyl phosphatidylcholine and palmitoyl-linoleoyl phosphatidylcholine. The saturated, long-chain phosphatidylcholine, dipalmitoyl phosphatidylcholine, was found to be hydrolysed also at smaller areas per molecule. In agreement with the experiments on the erythrocyte membrane, monolayer experiments show phospholipase $C(B$. cereus $)$ activity at pressures above 31 dynes $/ \mathrm{cm}$ after pretreatment of a phosphatidylcholine-sphingomyelin monolayer with sphingomyelinase ( $S$. aureus). The presence of neutral molecules as ceramide, the degradation product of sphingomyelinase action, enhance the breakdown at high pressures (Fig. 5). Diacylglycerol, the phospholipase $\mathrm{C}$ degradation product, also shows this effect. Differential scanning calorimetry and electron microscopy showed that these two neutral lipids do not form a homogeneous phase with phospholipids. For membranes showing phase separation, high ion permeability [11] and penetrability by pig pancreas phospholipase $A_{2}[12]$ is demonstrated. The high compressability at the phase borders or increased mobility of the polar headgroup could enhance phospholipase $\mathrm{C}$ (B. cereus) action. Due to electrostatic repulsion, positively and negatively charged lipids can increase the mean molecular area of neutral molecules. The increased molecular areas could explain the degradation of phosphatidylcholine in the presence of stearylamine and phosphatidic acid. The negative charge gives a higher stimulation than the positive charge. Bangham and Dawson [5] found that for the action of phospholipase $\mathrm{C}$ from $C$. perfringens a net positive charge at the interface is required. The stimulating effect of negatively charged lipids on the $B$. cereus phospholipase $C$ degradation explains also why this phospholipase, which cannot attack the intact cell, can hydrolyse lipids of the inner layer when trapped inside. The inner erythrocyte membrane consists mainly of phosphatidylethanolamine and phosphatidylserine in contrast to the outer layer which consists mainly of phosphatidylcholine and sphingomyelin. Monolayers of phosphatidylethanolamine/phosphatidylserine (molar ratio $2: 1)$ are hydrolysed at pressures of 39 dynes/ $\mathrm{cm}$ in contrast to pure phosphatidylethanolamine monolayers. Erythrocyte membranes contain a high content of unesterified cholesterol. The total phospholipid/sterol ratio is close to unity. In monomolecular films, the addition of $25 \mathrm{~mol} \%$ cholesterol to a mixture of phosphatidylcholine and sphingomyelin did not seem to have much effect on the enzymatic activity of phospholipase $\mathrm{C}$ from $B$. cereus. However, molar concentrations of $50 \%$ cholesterol in a phosphatidylcholine/sphingomyelin film show degradation also at high pressures. The latter result is difficult to correlate with the inability of $B$. cereus phospholipase $\mathrm{C}$ to hydrolyse phospholipids of the intact erythrocyte membrane. Until now there has been little experimental evidence about the distribution of cholesterol in the cell membrane and about the concentration in the outer layer. Recent ${ }^{31} \mathrm{P}$ NMR studies have indicated that in vesicles the ratio of cholesterol in the inner layer to cholesterol in the outer layer can be larger than 1 (de Kruyff, B., personal communication). Also proteins, interacting with cholesterol as demonstrated for the myelin membrane [13], could be feasible for the erythrocyte membrane. 
Phospholipase $C$ from $C$. welchii hydrolyses phosphatidylcholines as well as sphingomyelins. This enzyme is able to lyse erythrocytes due to the hydrolyses of both phospholipids. Monolayers of pure palmitoyl-oleoyl phosphatidylcholine are hydrolysed at pressures below 28 dynes $/ \mathrm{cm}$. However, sphingomyelin is hydrolysed also at high pressures. The behaviour of phospholipase $C$ from $C$. welchii towards monolayers correlates well with the action on erythrocytes since monolayers of phosphatidylcholine/sphingomyelin/cholesterol molar ratio $15: 12: 25$ and $15: 12: 9$ the phospholipids are hydrolysed at pressures above 31 dynes $/ \mathrm{cm}$. The low concentrations of cholesterol $(25 \mathrm{~mol} \%)$ enhance already the hydrolysis by phospholipase $C$ from C. welchii in monolayers by this for erythrocytes lytic enzyme. Besides the phospholipase $C$ from $B$. cereus also the phospholipases $\mathrm{A}_{2}$ from pig pancreas and $C r$.adamanteus are by themselves not able to hydrolyse phospholipids of the intact erythrocyte membrane. On monolayers, the two latter phospholipases show an even lower maximal pressure than phospholipase $\mathrm{C}$ from $B$. cereus. This means that the molecular pressure of the erythrocyte is too high for these phospholipases to penetrate and hydrolyse the lipid core. The phospholipases $\mathrm{A}_{2}$ which are able to hydrolyse membrane phospholipids, phospholipase $\mathrm{A}_{2}$ from $N$. naja and bee venom, show a maximal pressure above 31 dynes $/ \mathrm{cm}$. Several years ago Shah and Schulman [14] studied already the enzymatic hydrolysis of various phosphatidylcholine monolayers by $N$. naja venom, employing the surfacz pressure and potential technique. These authors found that the rate of hydrolysis of different phosphatidylcholines decreased in the same order as their molecular areas. The limiting pressure for egg lecithin was 30 dynes $/ \mathrm{cm}$ and for soybean lecithin 37 dynes $/ \mathrm{cm}$. The action of phospholipases on erythrocyte membranes and on monomolecular films is summarised in Table I. The phospholipases which cannot hydrolyse phospholipids of the intact erythrocyte membrane, phospholipase $\mathrm{C}$ from $B$. cereus, phospholipase $\mathrm{A}_{2}$ from pig pancreas and $C r$.adamanteus, phospholipase D from cabbage show maximal pressures below 31 dynes $/ \mathrm{cm}$. The phospholipases which can hydrolyse phospholipids of the intact erythrocyte membrane, phospholipase $\mathrm{C}$ from $C$. welchii, phospholipase $\mathrm{A}_{2}$ from $N$. naja and bee venom, sphingomyelinase from $S$. aureus, show maximal

\section{TABLE I}

COMPARISON OF THE EFFECTS OF DIFFERENT PHOSPHOLIPASES ON ERYTHROCYTE MEMBRANES AND THE MAXIMAL SURFACE PRESSURE AT WHICH THESE PHOSPHOLIPASES CAN HYDROLYSE MONOMOLECULAR FILMS

Phospholipases

Phospholipase $\mathrm{A}_{2}$ (pig pancreas)

$\mathrm{D}$ (cabbage)

$\mathrm{A}_{2}$ (Cr. adamantelts)

C (B. cereus)

$\mathrm{A}_{2}$ (N. naja)

$\mathrm{A}_{2}$ (bee venom)

Sphingomyelinase ( $S$. aureus)

Phospholipase C (C, welchii)

Phospholipase C (B. cereus) ,

Sphingomyelinase ( $S$. aureus)

\section{Erythrocytes}

Monolayers

(dynes/cm)

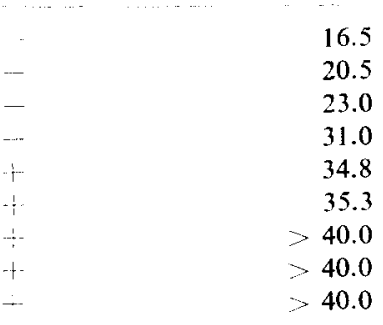


pressures above 31 dynes $/ \mathrm{cm}$. From the above results it can be concluded that the membrane pressure of the erythrocyte is likely to be between 31 and 34.8 dynes $/ \mathrm{cm}$. This is a higher lateral pressure within the bilayer membrane than suggested by Marčelja [15]. This author derived from order parameter calculations and NMR measurements a pressure of 20 dynes $/ \mathrm{cm}$. The experiments described in this paper, showing an excellent correlation between the action of phospholipases on monolayers and erythrocyte membranes, are a more direct measure of the lateral pressure. This pressure is close to the highest lateral pressure in monolayers.

In 1925 Gorter and Grendel [16] measured the area occupied by the lipids extracted from erythrocyte membranes, to estimate if the amount of lipid present is sufficient to cover the erythrocyte cell with a bilayer. Bar et al. [17] repeated these experiments and found that the ratio of monolayer film area to erythrocyte cell area depended greatly on the film pressure. A ratio of the film area to cell area of 2 is possible at a surface pressure of approx. 9 dynes $/ \mathrm{cm}$. The lateral film pressure of 31-34.8 dynes/cm suggested in this paper would lead to a ratio of film area to cell area of 1.3-1.7. This might implicate the remaining area of the cell membrane is occupied by intrinsic membrane proteins.

\section{REFERENCES}

1 Verkley, A. J., Zwaal, R. F. A., Roelofsen, B., Comfurius, P., Kastelijn, D. and van Deenen, L. L. M. (1973) Biochim. Biophys. Acta 323, 178-193

2 Zwaal, R. F. A., Roelofsen, B. and Colley, C. M. (1973) Biochim. Biophys. Acta 300, 159-182

3 Kahlenberg, A., Walker, C. and Rohrlick, R. (1974) Can. J. Biochem. 52, 803-806

4 Zwaal, R. F. A., Roelofsen, B., Comfurius, P. and van Deenen, L. L. M. (1975) Biochim. Biophys. Acta 406, 83-96

5 Bangham, A. D. and Dawson, R. M. C. (1962) Biochim. Biophys. Acta 59, 103-115

6 Colacicco, G. and Rapport, M. M. (1965) Fed Proc. 24, 295-301

7 Miller, I. R. and Ruysschaert, J. M. (1971) J. Coll. Interface Sci. 35, 340-345

8 Verger, R. and de Haas, G. H. (1973) Chem. Phys. Lipids 10, 127-136

9 Stoffel, W., Lekim, D. and Tschung, T. S. (1971) Hoppe Seyler's Z. Physiol. Chem. 352, 1058-1062

10 Low, M. G., Limbrick, A. R. and Finean, J. B. (1973) FEBS Lett. 34, 1-4

11 Haest, C. W. M., de Gier, J., van Es. G. A., Verkley, A. J. and van Deenen L. L. M. (1972). Biochim. Biophys. Acta 288, 43-53

12 Op den Kamp, J. A. F., de Gier, J. and van Deenen, L. L. M. (1974) Biochim Biophys. Acta $345,253-256$

13 London, Y., Demel, R. A., Geurts van Kessel, W. S. M., Zahler, P. and van Deenen, L. L. M. (1974) Biochim. Biophys. Acta 332, 69-84

14 Shah, D. O. and Schulman, J. H. (1967) J. Coll. Interface Sci. 25, 107-119

15 Marčelja, S. (1974) Biochim. Biophys. Acta 367, 165-176

16 Gorter, E. and Grendel, F. (1925) J. Exp. Med. 41, 439-446

17 Bar, R. S., Deamer, D. W. and Cornwell, D. G. (1966) Science 153, 1010-1012 